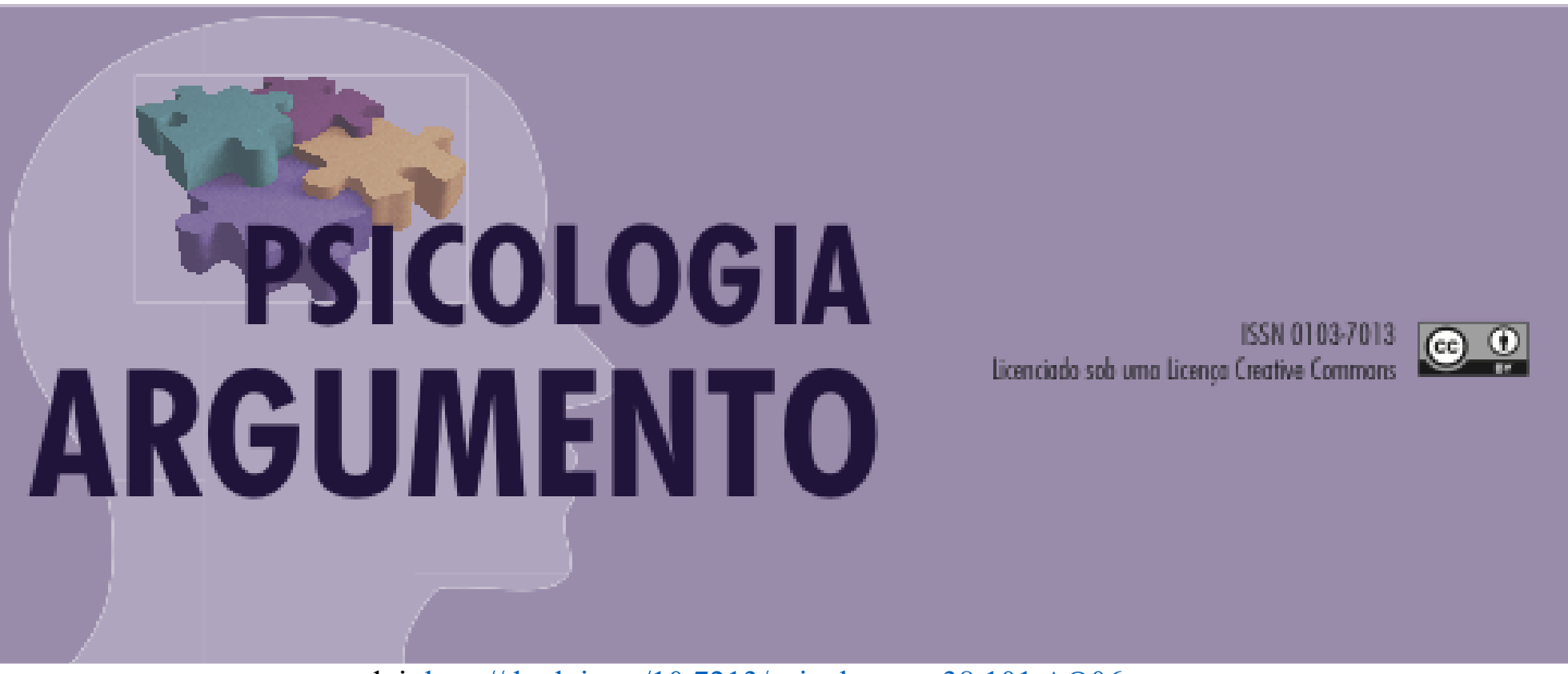

doi: http://dx.doi.org/10.7213/psicolargum.38.101.AO06

\title{
Escala de Comportamentos Antissociais: construção e estudos psicométricos
}

Antisocial Behavior Scale: construction and psychometrics studies

Escala de Comportamientos Antisociales: construcción y estudios psicométricos

Ana Deyvis Santos Araújo Jesuíno

Centro de Ciências e Tecnologias do Maranhão (UniFacema), e-mail: anadeyvis@gmail.com, http://orcid.org/0000-0002-7031-7682

Fabián Javier Marín Rueda

Universidade São Francisco - USF e Centro Universitário de Brasilia (UniCEUB), e-mail: fabian.rueda@usf.edu.br, http://orcid.org/0000-0001-5173-0802

\section{Resumo}

O objetivo deste estudo foi descrever a construção e os estudos psicométricos iniciais de uma escala para avaliar o comportamento antissocial. Foram realizados estudos de avaliação de itens por juízes, estudo piloto para verificar a interpretabilidade dos itens e posteriormente os estudos psicométricos com 600 pessoas dos estados do Piauí, São Paulo e Curitiba. A análise dos itens indicou um modelo com um bom índice de ajuste. O modelo é formado cinco fatores denominados agressividade, irresponsabilidade, manipulação interpessoal, comportamento de risco no trânsito e inconsequência. Os valores de fidedignidade encontrados foram adequados. Os resultados permitiram concluir que os objetivos iniciais foram alcançados.

Palavras-chave: avaliação psicológica; antissocial; teste psicológico; psicometria 


\begin{abstract}
This study aimed to describe the construction and initial psychometric studies of an instrument to assess antisocial behavior. Studies were the evaluation of the items by judges, a pilot study to verify the interpretability of the items and, subsequently, psychometric studies with 600 people from the states of Piaui, São Paulo, and Curitiba. The analysis of the items indicated a model with good-fit indexes. The model consists of five factors called aggressiveness, irresponsibility, interpersonal manipulation, risk behavior in traffic and inconsequence. The reliability values were adequate. The results allowed us to conclude that we achieved the initial objectives.
\end{abstract}

Keywords: psychological assessment; antisocial; psychological test; psychometric

\title{
Resumen
}

El objectivo de esto estudio fue describir la construcion y estudios psicometricos iniciales de uma escala para evaluar comportamiento antisocial. Los itens fueran evaluados por juices, despues verifcaran la interpretabilidad dos itens y los estudios psicometricos com 600 personas de provincias del Piauí, São Paulo e Curitiba. La analises de los itens indicó um modelo con um bueno ajuste. El modelo és formado por cinco factores denominados agressividad, irresponsabilidad, manipulacion interpersonal, comportamiento de risgo em trânsito $y$ inconsecuencia. La fidedignidad fue adecuada. Los resultados permitiran concluier que los objectivos iniciales fueran alcanzados.

Palabras clave: evaluacion psicológica, antisocial, teste psicológico, psicometria

\section{Introdução}

O comportamento antissocial (CAS) é caracterizado principalmente pela violação do direito de outras pessoas, envolvimento em atividades delitivas (Donavan \& Jessor, 1985) como roubo, vandalismo, condução imprudente e instabilidade de emprego, entre outros aspectos. Desvios de conduta relacionados a falta de controle de impulsos ou a comportamentos hostis podem estar presentes (Derefinko \& Widiger, 2016; Santana et al., 2018) . Também pode ser compreendido por um componente de tendências agressivas (objetivo de causar algum dano físico ou psicológico a alguém ou grupo de pessoas), especificamente reativas (respostas a provocações e ocorrem de forma impulsiva como uma defesa associada a raiva e frustração) e proativas (uma antecipação a respostas dos outros, é voluntário, deliberado e influenciado por reforços externos) [(Burt, Donnellan, $\&$ Tackett, 2012)].

A utilização de modelos básicos da personalidade pode contribuir consideravelmente para a compreensão do CAS. Uma metanálise que objetivou verificar a relação entre os diferentes modelos teóricos da personalidade e o CAS, e identificou o 
comportamento interpessoal antagônico e um estilo impulsivo cognitivo e comportamental como maiores preditores (Miller \& Lynam, 2001).

Esses fatos indicam que o comportamento antissocial não está necessariamente ligado a algo patológico, como um transtorno da personalidade, mas pode vir a ser, pois é considerado um aspecto dimensional, entendido como um continuum e pode ser avaliado por meio de entrevistas semiestruturadas e testes de autorrelato (Marcus, Lilienfeld, Edens, \& Poythress, 2006). A presença extrema desse traço caracteriza o Transtorno da Personalidade Antissocial (American Psychiatric Association- APA, 2014; Kotov, 2017).

$\mathrm{Na}$ quinta versão do Manual Diagnóstico e Estatístico de Transtornos Mentais (Diagnostic and Statistical Manual of Mental Disorders - DSM 5) é apresentado um modelo alternativo para avaliação da personalidade por meio de traços compreendidos como um continuum. De acordo com esse modelo o comportamento antissocial e o transtorno de personalidade antissocial podem ser avaliados com base nos domínios de desinibição (gratificação imediata desconsiderando o aprendizado passado ou consequências futuras) e antagonismo (comportamentos interpessoais divergentes, que incluem um sentimento exagerado da própria importância e antipatia insensível em relação aos outros) (APA, 2014) o que também é compartilhado por outro modelo diagnóstico denominado Hierarchical Taxonomy of Psychopathology (HiTOP) (Conway et al., 2018; Forbes, et al., 2017; Kotov et al., 2017).

A desinibição engloba os traços Exposição ao risco, a Impulsividade e a Irresponsabilidade enquanto a manipulação diz respeito aos traços de Manipulação, Insensibilidade, Desonestidade e Hostilidade. A Exposição ao Risco refere-se ao envolvimento em atividades arriscadas, de perigo iminente para tentar contrapor ao tédio. A impulsividade diz respeito a uma ação imediata sem considerar as consequências. A irresponsabilidade refere-se ao descumprimento de compromissos financeiros e de obrigações assumidas. Em relação aos traços antagônicos, o primeiro citado é a Manipulação que diz respeito a capacidade de influenciar outras pessoas para obter algum benefício. A Insensibilidade por sua vez, é a falta de preocupação com os sentimentos ou problemas de outras pessoas. A Desonestidade está relacionada a fraudes e a Hostilidade a raiva ou irritabilidade e comportamentos grosseiros (APA, 2014).

Um estudo realizado anteriormente verificou a estrutura fatorial dos critérios do DSM-IV e se de fato são necessários distintos fatores para avaliação do transtorno da 
personalidade antissocial (Kendler, Aggen, \& Patrick, 2012). Foram analisados além dos critérios fatores fenotípicos em gêmeos. Os autores encontraram dois fatores correlacionados que avaliam padrões de agressividade e desprezo e outro de desinibição. Pontuações no escore genético de agressividade e desprezo estavam mais associadas ao risco para transtorno de conduta e o uso de álcool, enquanto a desinibição estava associada a idade e busca de sensações. Recentemente Rosenström, et al. (2018) também realizou um estudo com gêmeos e a entrevista semiestruturada do DSM-IV. Os autores encontraram um modelo de fator geral de segunda ordem que explicava os critérios diagnósticos. Isso indica que ainda existem divergências a respeito da melhor estrutura avaliativa para aspectos relacionados ao comportamento antissocial.

O objetivo do presente artigo foi construir uma escala para avaliar o comportamento antissocial considerando a agressividade reativa e proativa (Burt et al., 2012) e as dimensões do antagonismo e da desinibição (APA, 2014). Para tanto foram utilizados os traços dos dois domínios, pois como evidenciado anteriormente (Jones, Miller \& Lynam, 2011) a relação entre o comportamento antissocial e outros traços de personalidade foi mais consistente por meio de facetas, como por exemplo, impulsividade e hostilidade, e não por meio dos domínios. Para além disso, considerou-se para a construção os especificadores da Psicopatia como descrito no DSM-5, que dizem respeito a ausência de ansiedade ou medo, audácia e busca por atenção.

\section{Objetivos}

Construir e realizar estudos psicométricos iniciais para a Escala de Avaliação de Comportamentos Antissociais (E-Cant).

\section{Método}

\section{Estudo 1- Etapa 1- Elaboração dos Itens e avaliação de juízes}

O processo de construção levou em consideração as definições apresentadas na Seção III do DSM-5 para os traços dos domínios desinibição e antagonismo. Os itens foram construídos pela autora deste trabalho a partir da descrição dos fatores Manipulação, Hostilidade, Desonestidade e Insensibilidade (Antagonismo) e Irresponsabilidade, Impulsividade, Exposição ao risco (Desinibição). Os itens de agressividade foram construídos considerando a agressividade reativa e proativa (Coie \& Dodge, 1998). Além disso também foram construídos itens para os especificadores da 
psicopatia. Inicialmente foram construídos um total de 110 itens distribuídos entre 8 a 12 itens para cada fator (Manipulação, Insensibilidade, Desonestidade, Hostilidade, Exposição ao risco, Irresponsabilidade, Impulsividade, Agressividade Reativa e Proativa ou instrumental e para os descritores de Psicopatia). Cuidou-se para que os itens não apresentassem conteúdo similar e para tanto foram realizadas quatro revisões no conteúdo dos mesmos o que resultou em 88 itens.

\section{Participantes}

Participaram desta etapa cinco psicólogos, sendo três do sexo masculino, do estado de São Paulo. Todos são doutores em psicologia com conhecimento em construção de testes e em avaliação psicológica da personalidade.

\section{Instrumento}

Os autores construíram um protocolo de avaliação que foi enviado aos psicólogos. Eles deveriam indicar o nível de clareza e linguagem, possíveis correções ortográficas, a relevância teórica e os fatores ao qual pertenciam cada um dos 88 itens além de informar o modelo de resposta Likert que deveria ser adotado.

\section{Procedimentos Gerais}

Inicialmente foi solicitada a autorização para as instituições para a realização da coleta. Após a autorização o projeto foi enviado ao Comitê de Ética em Pesquisa da Universidade São Francisco. Após sua aprovação (CAEE: 69599717.1.0000.5514) as coletas foram agendadas e realizadas considerando cada etapa do processo de construção. Os sujeitos foram informados, em todas as etapas, do objetivo da pesquisa. Após lerem e assinarem o Termo de Consentimento Livre e Esclarecido (TCLE) realizaram a atividade pertinente em cada etapa.

\section{Procedimentos Etapa 1}

Os juízes foram convidados por e-mail para esta avaliação, sendo que após o aceite receberam o arquivo com os itens e uma explicação breve sobre a teoria utilizada para a construção dos mesmos e as definições dos fatores. Para a participação foi necessário que os mesmos concordassem TCLE. Os juízes tiveram um prazo de um mês para enviarem suas repostas sobre o quanto os itens descritos avaliavam Manipulação, Insensibilidade, Hostilidade, Desonestidade, Exposição ao Risco, Impulsividade, Irresponsabilidade Agressividade reativa e proativa e os descritores da psicopatia. 


\section{Análise de dados}

Os protocolos de resposta foram analisados com base nas sugestões dos juízes, desde modificações, categorização, a remoção e divisão de itens. Foi utilizado como critério para a manutenção do item no teste uma concordância mínima de $75 \%(p<0,05)$, equivalente ao consentimento de 3 juízes.

\section{Resultados Etapa 1}

Após a avaliação semântica, relevância teórica e categorização dimensional foram excluídos um total de 24 itens e a chave de resposta escolhida foi a de 4 pontos ( $1=$ nada a ver comigo a 4=tudo a ver comigo). Um deles apresentava exemplos de situações "Gosto de atividades que geram adrenalina (como saltar de paraquedas, andar em alta velocidade)" foi dividido ficando cada exemplo em um item diferente. Em relação a dimensão Antagonismo o fator Manipulação (1) ficou composto por 8 itens, o fator Insensibilidade (2) com um total de 6 itens, Desonestidade (3) com 5 itens e Hostilidade (4) com 5 itens. No que diz respeito a dimensão Desinibição, foram categorizados 9 itens no fator (5) Exposição ao Risco. O fator Irresponsabilidade (6) foi composto por 9 itens, Impulsividade (7) com 3 itens e agressividade reativa e proativa com 14 itens ao total e os indicadores de psicopatia com cinco itens. No total ficaram 64 itens com modelo de resposta do tipo Likert de 4 pontos em que 1 é nada a ver comigo, 2 é pouco a ver comigo, 3 é muito a ver comigo e 4 é tudo a ver comigo. Finalizada a primeira etapa deram-se início as coletas.

\section{Estudo 1 Etapa 2 - Estudo Piloto}

Objetivo: Verificar a compreensão dos itens por pessoas de baixa escolaridade Participantes

A etapa seguinte consistiu na avaliação dos itens por 5 pessoas, sendo 3 mulheres, do estado do Piauí, com nível de escolaridade do ensino fundamental ambos os sexos e idade entre 33 e 54 anos. Esta foi realizada para certificar que os itens pudessem ser compreendidos por pessoas de diferentes níveis de escolaridade.

\section{Instrumentos}

Escala de Comportamentos Antissociais (E-Cant)

$\mathrm{O}$ instrumento contou com um questionário de identificação que visou obter informações sobre sexo, idade, escolaridade, cidade, entre outros. Foi utilizada a versão 
com itens que foram avaliados pelos juízes e por fim, o questionário sobre dificuldades de compreensão dos itens.

\section{Procedimentos}

Foi solicitada a autorização da instituição para a realização da pesquisa. Após uma explicação sobre o objetivo da pesquisa e a assinatura do TCLE os participantes responderam o protocolo. A aplicação foi coletiva com 5 indivíduos e durou cerca de 15 minutos.

\section{Resultados}

Após responderem os itens os participantes não relataram dificuldades para compreender o conteúdo utilizado. Dessa forma, considerou-se que os itens estavam adequados para pessoas com baixo nível de escolaridade e consequentemente para aqueles com maior nível.

\section{Estudo 2 - Verificação das Propriedades Psicométricas}

\section{Participantes}

Participaram 600 indivíduos, sendo 54,4\% mulheres, com idade entre 18 a 80 anos $(M=30,50 ; D P=12,91)$. A seleção dos participantes foi realizada de forma aleatória. A maioria deles universitários, com ensino superior completo/incompleto $(66,1 \%)$ e residentes dos estados do Piauí (35\%) e São Paulo (32,3\%). Os outros participantes residiam em Curitiba (32,6\%) e foram recrutados no Departamento Nacional de Trânsito (DETRAN) do estado do Paraná.

\section{Instrumento}

\section{Escala de Comportamentos Antissociais (E-Cant)}

Versão de 64 itens apresentada nos resultados da Etapa 1 do estudo 1.

\section{Procedimentos}

A coleta foi agendada e os sujeitos foram informados do objetivo da pesquisa. Após esclarecimentos sobre a mesma e a assinatura do TCLE deu-se início a coleta de dados. Os participantes responderam a um questionário sociodemográfico e a E-Cant. A aplicação durou aproximadamente 15 minutos com cerca de 30 pessoas por aplicação. 


\section{Análise de dados}

Inicialmente foram realizadas as estatísticas descritivas. A fatorabilidade da matriz foi verificada por meio do critério de Kaiser-Meyer-Olkin (KMO) e do Teste de Esfericidade de Bartlett. Posteriormente, foi realizada a Análise Fatorial Exploratória (AFE) por meio do programa Factor 10.8 (Lorenzo-Seva \& Ferrando, 2018) com metade dos participantes escolhidos aleatoriamente no banco de dados (301 sujeitos). O procedimento para determinar o número de dimensões foi a Parallel Analysis (PA), com matriz de dispersão Polychoric Correlations e como método com rotação do tipo Promim (Lorenzo-Seva \& Ferrando, 2018). Foram mantidos nos fatores apenas os itens com carga fatorial superior a 0,50 consideradas boas. Para itens que carregaram em dois fatores foi considerada uma diferença mínima de 0,20 entre eles bem como a pertinência teórica. Posteriormente foi utilizada a outra parte do banco para testar modelos restritivos e saturados. O estimador utilizado foi Weighted Least Squares Mean- and Varianceadjusted (WLSMV) que é o mais indicado para dados ordinais (Asún, Rdz-Navarro, \& Alvarado, 2016), com rotação do tipo oblimin sendo estas ultimas realizadas no programa Mplus 7.10 (Muthén \& Muthén,1998-2012). O ajuste dos modelos foi verificado pelos índices Chi-square $\left(\chi^{2}\right)$, Comparative Fit Index (CFI), Tucker-Lewis Index (TLI) e Root Mean Square Error of Approximation (RMSEA). Por fim, o nível de fidedignidade foi verificado utilizando o coeficiente alfa de Cronbach.

\section{Resultados}

O valor de KMO encontrado foi de 0.870 , considerado bom, e do Teste de Esfericidade de Bartlett foi de 8275.6 .3 ( $d f=2016$; $p<0.001)$ indicando os dados são passíveis de fatoração (Dziuban \& Shirkey, 1974). A análise paralela indicou uma estrutura fatorial de 7 fatores. Foram testados modelos de 5, 6, 7 e oito fatores com os 64 itens. O modelo de 6 fatores foi o mais explicável. O ponto de corte para a exclusão de itens foi de 0,30 .

Uma nova análise fatorial exploratória foi realizada com os 57 itens utilizando os mesmos métodos de extração do modelo inicial para 5 e 6 fatores. Ao realizar tal etapa os itens não apresentaram bons índices de ajuste. Uma nova análise foi realizada com o modelo de 5 fatores considerando os 64 itens e ao verificar as cargas fatoriais foram excluídos mais 16 itens com carga inferior ao ponto estabelecido e os índices de ajustes 
encontrados foram considerados bons com $\chi^{2} \quad(898)=1268.969^{*} \quad(p<0,001)$, RMSEA=0,037 (90\% IC: $0.032-0.042 ; p<0,05), \mathrm{CFI}=0,951$ e TLI=0,93. Os valores das cargas fatoriais foram altas e podem ser visualizadas na Tabela 1.

Tabela 1.

Cargas fatoriais e quantidade de itens

\begin{tabular}{|c|c|c|c|c|c|}
\hline Itens & F1 & F2 & F3 & F4 & F5 \\
\hline E1 & 0.065 & $0.175^{*}$ & 0.099 & 0.056 & $0.286^{*}$ \\
\hline E2 & -0.006 & $0.230 *$ & $0.480 *$ & -0.014 & 0.022 \\
\hline E3 & 0.107 & 0.024 & 0.083 & $0.358 *$ & $-0.204^{*}$ \\
\hline E6 & $0.802 *$ & $-0.125^{*}$ & 0.015 & 0.032 & -0.009 \\
\hline E7 & 0.040 & $0.841 *$ & 0.029 & 0.006 & -0.070 \\
\hline E8 & $-0.378 *$ & 0.002 & 0.119 & 0.124 & $0.216^{*}$ \\
\hline E9 & $-0.222 *$ & $0.443 *$ & $0.189^{*}$ & $0.219^{*}$ & 0.059 \\
\hline E10 & -0.003 & 0.056 & -0.036 & $0.733 *$ & $0.180 *$ \\
\hline E14 & 0.045 & $0.659 *$ & -0.049 & $-0.149 *$ & 0.045 \\
\hline E17 & $0.214^{*}$ & $0.222 *$ & $0.452 *$ & -0.045 & 0.071 \\
\hline E18 & $0.503 *$ & -0.055 & $0.203^{*}$ & $-0.164 *$ & 0.054 \\
\hline E19 & $-0.187 *$ & $0.774 *$ & 0.056 & $0.157 *$ & -0.006 \\
\hline E20 & 0.104 & $0.568 *$ & -0.046 & 0.029 & 0.075 \\
\hline E21 & $0.790 *$ & 0.055 & $-0.175^{*}$ & -0.050 & -0.053 \\
\hline E22 & 0.019 & 0.034 & $0.496 *$ & $0.497 *$ & -0.109 \\
\hline E23 & $0.218^{*}$ & -0.011 & -0.120 & $0.708 *$ & -0.067 \\
\hline E24 & $0.142 *$ & 0.100 & 0.470 * & -0.064 & $0.203^{*}$ \\
\hline E25 & 0.101 & -0.040 & $0.526 *$ & $0.511 *$ & $-0.145^{*}$ \\
\hline E26 & $0.142 *$ & $0.167 *$ & $0.532 *$ & $0.147^{*}$ & -0.126 \\
\hline E27 & $0.177^{*}$ & $0.252 *$ & $0.480 *$ & 0.050 & $0.120 *$ \\
\hline E28 & $0.578 *$ & 0.003 & 0.126 & $0.169^{*}$ & 0.091 \\
\hline E29 & 0.809* & 0.016 & 0.056 & $-0.147 *$ & -0.010 \\
\hline E30 & $0.165^{*}$ & $0.211^{*}$ & $0.640 *$ & 0.001 & -0.007 \\
\hline E31 & 0.066 & 0.065 & $0.604 *$ & $0.226^{*}$ & 0.052 \\
\hline E33 & 0.071 & $0.864 *$ & -0.031 & -0.052 & -0.018 \\
\hline E35 & 0.078 & $0.160 *$ & $0.728 *$ & -0.019 & -0.019 \\
\hline E36 & -0.007 & -0.021 & $0.861 *$ & $-0.123^{*}$ & $0.160 *$ \\
\hline E37 & $0.723 *$ & -0.029 & 0.098 & $0.165^{*}$ & 0.073 \\
\hline E38 & $0.680 *$ & 0.008 & $0.304^{*}$ & $0.157^{*}$ & 0.035 \\
\hline E39 & $0.850 *$ & 0.084 & $-0.099 *$ & 0.007 & 0.007 \\
\hline $\mathrm{E} 40$ & 0.034 & 0.086 & -0.057 & $0.670 *$ & $0.255^{*}$ \\
\hline E41 & -0.015 & $0.745^{*}$ & $0.175^{*}$ & -0.012 & 0.023 \\
\hline E43 & $0.528 *$ & -0.104 & $0.157^{*}$ & 0.078 & $0.150 *$ \\
\hline E44 & $0.677 *$ & -0.021 & 0.121 & 0.139 & $-0.137^{*}$ \\
\hline $\mathrm{E} 45$ & 0.055 & -0.019 & 0.004 & 0.031 & $0.767 *$ \\
\hline E46 & $0.769 *$ & 0.018 & -0.091 & $0.220 *$ & 0.060 \\
\hline E49 & $0.732 *$ & $0.112 *$ & 0.000 & -0.052 & -0.010 \\
\hline E50 & $0.181 *$ & 0.108 & $0.561 *$ & 0.068 & -0.005 \\
\hline E52 & 0.067 & $0.815 *$ & $-0.087^{*}$ & -0.024 & $0.129 *$ \\
\hline E53 & $0.661 *$ & $0.136^{*}$ & 0.022 & -0.035 & 0.012 \\
\hline E54 & 0.078 & 0.109 & 0.086 & $0.185^{*}$ & $0.645^{*}$ \\
\hline E55 & $0.760 *$ & 0.034 & 0.075 & $-0.134^{*}$ & $0.101 *$ \\
\hline E57 & $0.450 *$ & 0.158 & 0.120 & $0.258 *$ & 0.012 \\
\hline E58 & -0.069 & $-0.119 *$ & 0.023 & 0.100 & $0.677 *$ \\
\hline E60 & 0.024 & 0.619* & $0.133^{*}$ & 0.100 & -0.058 \\
\hline E61 & $-0.146^{*}$ & $-0.154^{*}$ & $0.658 *$ & -0.041 & $0.207 *$ \\
\hline E62 & 0.035 & 0.094 & 0.051 & -0.084 & $0.756 *$ \\
\hline E63 & 0.098 & 0.139 & $0.180^{*}$ & $0.471 *$ & $0.086-$ \\
\hline $\mathrm{N}^{0}$ de itens & 16 & 9 & 13 & 7 & 4 \\
\hline
\end{tabular}


Com base na tabela 1 é possível verificar que o item 1 não carregou em nenhum dos fatores e que o item 22 carregou em dois fatores. Considerando o conteúdo dos itens e a importância deles para o modelo fatorial optou-se por mantê-los e realizar uma nova análise para verificar modelos restritivos e saturados. Pode-se observar também que o fator 1 agrupou itens que avaliam, em sua maioria, agressividade (10 itens) e hostilidade (4 itens) e um item que avalia insensibilidade. O Fator 2 agrupou itens que avaliam a irresponsabilidade, enquanto o fator 3 foi formado por itens que avaliam descritores da psicopatia, especificamente de necessidade de atenção (3 itens) e itens de desonestidade (2 itens) e manipulação ( 8 itens) denominado manipulação interpessoal. Por sua vez, o fator 4 foi composto por comportamento de risco no trânsito e o fator 5 por itens que avaliam a exposição ao risco (4 itens).

Posteriormente foram testados os modelos restritivos e saturados. A análise desse modelo permitiu a exclusão de 3 itens (E8, E38 e E54). Os índices de ajuste do modelo foram bons com valores de $\chi^{2}(835)=1480.650^{*}(p<0,001)$, RMSEA=0,044 (95\% IC: $0.040-0.048), \mathrm{CFI}=0,921$ e $\mathrm{TLI}=0,917$.

Inicialmente optou-se por verificar o modelo bifator, contudo o modelo não se ajustou aos dados pois as cargas fatoriais não carregaram no modelo geral. Posteriormente realizou-se a verificação de um modelo hierárquico considerando dois fatores de ordem superior. Os índices de ajuste encontrados foram bons com valores de $\chi^{2}(659)=$ $1628.553 *(p<0,001)$, RMSEA=0,063 (95\% IC: 0.059 - 0.067), CFI $=0,970$ e TLI $=0,969$. As cargas fatoriais variaram de 0,40 a 0,88 . As análises dos alfas indicaram um alfa total de 0,92 e entre os fatores variou de 0,65 a 0,87. Cabe destacar que o valor não aumentava com a exclusão de nenhum item. As cargas fatoriais de cada fator podem ser observadas na Tabela 2. 
Tabela 2.

Cargas fatoriais e fatores do modelo

\begin{tabular}{|c|c|c|c|c|c|}
\hline Item & $\begin{array}{l}\text { F1- } \\
\text { Agressividad } \\
\mathrm{e}(\alpha=0,89)\end{array}$ & $\begin{array}{l}\text { F2- } \\
\text { Irresponsabilid } \\
\text { ade }(\alpha=0,83)\end{array}$ & $\begin{array}{l}\text { F3-Manipulação } \\
\text { Interpessoal } \\
(\alpha=0,87)\end{array}$ & $\begin{array}{l}\text { F4-Comp. De Risco } \\
\text { no Trânsito }(\alpha=0,72)\end{array}$ & $\begin{array}{l}\text { F5- } \\
\text { Inconseque } \\
\operatorname{ncia}(\alpha=0,7 \\
\text { 2) }\end{array}$ \\
\hline E6 & 0.717 & & & & \\
\hline E18 & 0.532 & & & & \\
\hline E21 & 0.642 & & & & \\
\hline E28 & 0.759 & & & & \\
\hline E29 & 0.673 & & & & \\
\hline E37 & 0.845 & & & & \\
\hline E39 & 0.818 & & & & \\
\hline E43 & 0.639 & & & & \\
\hline E44 & 0.740 & & & & \\
\hline E46 & 0.815 & & & & \\
\hline E49 & 0.744 & & & & \\
\hline E53 & 0.727 & & & & \\
\hline E55 & 0.771 & & & & \\
\hline E57 & 0.769 & & & & \\
\hline E7 & & 0.849 & & & \\
\hline E9 & & 0.537 & & & \\
\hline E14 & & 0.583 & & & \\
\hline E19 & & 0.721 & & & \\
\hline E20 & & 0.640 & & & \\
\hline E33 & & 0.844 & & & \\
\hline E41 & & 0.847 & & & \\
\hline E52 & & 0.813 & & & \\
\hline E60 & & 0.748 & & & \\
\hline E2 & & & 0.585 & & \\
\hline E17 & & & 0.721 & & \\
\hline E22 & & & 0.746 & & \\
\hline E24 & & & 0.638 & & \\
\hline E25 & & & 0.770 & & \\
\hline E26 & & & 0.728 & & \\
\hline E27 & & & 0.815 & & \\
\hline E30 & & & 0.831 & & \\
\hline E31 & & & 0.771 & & \\
\hline E35 & & & 0.794 & & \\
\hline E36 & & & 0.727 & & \\
\hline E50 & & & 0.746 & & \\
\hline E61 & & & 0.425 & & \\
\hline E3 & & & & 0.404 & \\
\hline E10 & & & & 0.734 & \\
\hline E23 & & & & 0.675 & \\
\hline E40 & & & & 0.759 & \\
\hline E63 & & & & 0.889 & \\
\hline E1 & & & & & 0.702 \\
\hline E45 & & & & & 0.688 \\
\hline E58 & & & & & 0.501 \\
\hline E62 & & & & & 0.828 \\
\hline
\end{tabular}

Por meio da Tabela 2 pode-se observar uma variação de cargas fatoriais e que um fator abarcou apenas itens do trânsito. Em virtude disso realizou-se uma nova analise fatorial confirmatória para verificar a influência desses itens ao modelo e se o uso do mesmo era necessário. Os resultados indicaram que o modelo não sofreu alterações e 
apresentou bons índices de ajuste $\chi^{2}(734)=1247.810^{*}(p<0,001)$, RMSEA $=0,048(95 \%$ IC: $0.044-0.053), C F I=0,928$ e TLI=0,923. Esses valores foram semelhantes ao realizado com a inclusão de itens do trânsito e as cargas fatoriais apresentaram valores acima de 0.40 .

\section{Discussão}

A avaliação do Comportamento Antissocial tem como base traços desinibidos e antagônicos, incluindo agressividade, bem como especificadores da psicopatia como pressuposto por diferentes grupos de pesquisa (APA, 2014; Burt et al., 2012; Kotov et al., 2017). Para tanto, o objetivo principal deste artigo foi construir uma escala de comportamentos antissociais e verificar suas propriedades psicométricas iniciais.

Os resultados indicaram um modelo hierárquico parcimonioso com bons índices de ajuste para um conjunto de 45 itens com altas cargas fatoriais que indicam a alta capacidade discriminativa dos mesmos (Asún, et. al, 2016). Em amostras acima de 250 pessoas é esperado que valores de $\chi^{2}$ apresentem valores- $p$ significantes, CFI ou TLI acima de 0,90 e RMSEA $<0,07$. Os valores de alfa do instrumento foram considerados de bons a excelentes (Hair, Black, Babin, Anderson, \& Tatham, 2009). A quantidade de fatores extraídos do modelo pode ser compreendida ao se considerar que os traços, apesar de pertencerem a dimensões distintas que compartilham conteúdos similares. como é o caso de Hostilidade e Agressividade, que para alguns autores (e.g.: Lobbestael, Cima, \& Arntz, 2013), a hostilidade é preditora da agressividade. A Exposição ao risco, Impulsividade também partem do pressuposto de gratificações imediatas sem levar em consideração o aprendizado passado ou consequências futuras e estão associados a comportamentos irresponsáveis (APA, 2014). Por sua vez, a manipulação envolve engano e/ou coerção para com os outros (desonestidade), de forma intencional e planejada e a falta de empatia para com os outros (insensibilidade). Esses aspectos contribuem para a compreensão de agrupamentos de itens que ocorreram entre os fatores.

Apesar da relevância do estudo desenvolvido por Hauck-Filho et al. (2014), este estudo contrapõe o resultado encontrado pelos autores anteriormente citados pois parte de uma avaliação de múltiplos traços a partir dos domínios de desinibição e antagonismo como indicado por modelos avaliativos (APA, 2014; Conway et al., 2018; Kotov et al., 2017). Isso pode ser compreendido pela análise qualitativa dos itens, pois como apresentado pelos autores (Hauck-Filho et al., 2014) os itens do instrumento breve de 
comportamento antissocial descrevem antagonismo, baixa cooperação, hostilidade e agressão que são traços que avaliam primordialmente aspectos antagônicos o que justifica a unidimensionaliade encontrada pelos autores.

Em relação ao modelo estrutural encontrado um importante aspecto deve ser mencionado. Foram consideradas as duas dimensões, desinibição e antagonismo (APA, 2014; Kender, et al., 2012; Kotov, 2017), incluindo agressividade (Burt et al., 2012) para a construção do instrumento. Os resultados indicaram um bom ajuste para um modelo composto por cinco fatores que podem ser aplicados com os itens do trânsito quando se pretender avaliar especificamente motoristas, bem como a não utilização dos referidos itens.

\section{Considerações finais}

Apesar dos resultados aqui encontrados indicarem a E-Cant como um instrumento de qualidade é necessário indicar como limitação a baixa quantidade de itens sobre impulsividade que ficaram ao final. Nesse sentido é necessário que novos estudos sejam realizados com novos itens para verificar se a estrutura fatorial é mantida com a inserção de novos itens. Além disso, apesar de ter sido criada inicialmente para a população geral é necessário que estudos futuros sejam realizados considerando diferentes populações como a carcerária com homicidas e a relação com componentes psicossociais, condutores e pessoas com transtornos de personalidade que possuem o comportamento antissocial como característica chave. Pode-se pensar ainda em verificar um perfil característico do sujeito que apresentam tais comportamentos.

\section{Referências}

American PsychiatricAssociation ([APA]), (2014). Manual diagnóstico e estatístico de Transtornos Mentais -DSM-5.(5 edição). Porto Alegre: Artmed.

Asún, R. A., Rdz-Navarro, K., \& Alvarado, J. M. (2016). Developing multidimensional Likert scales using item factor analysis: The case of four-point items. Sociological Methods \& Research, 45(1), 109-133. doi: 10.1177/0049124114566716.

Burt, S. A., Donnellan, M. B., \& Tackett, J. L. (2012). Should social aggression be considered "antisocial"? Journal of Psychopathology and Behavioral Assessment, 34(2), 153-163. doi:10.1007/s10862-011-9267-0

Carey, G., \& Goldman, D. (1997). The genetics of antisocial behavior. In D. M. Stoff, J. Breiling, \& J. D. Maser (Eds.), Handbook of antisocial behavior (pp. 243-254). Hoboken, NJ, US: John Wiley \& Sons Inc. 
Coie, J. D., \& Dodge, K. A. (1998). Aggression and antisocial behavior. In W. Damon \& N. Eisenberg (Ed.), Handbook of child psychology: Social, emotional, and personality development (pp. 779-862). Hoboken, NJ, US: John Wiley \& Sons Inc.

Conway, C., Forbes, M. K., Forbush, K. T., Fried, E. I., Hallquist, M. N., Kotov, R., ... Eaton, N. R. (2018). A Hierarchical Taxonomy of Psychopathology Can Transform Mental Health Research. doi:10.17605/OSF.IO/WSYGP

Derefinko, K. J., \& Widiger, T. A. (2016). Antisocial personality disorder. In The medical basis of psychiatry (pp. 229-245). New York, NY: Springer.

Donovan, J. E., \& Jessor, R. (1985). Structure of Problem Behavior in Adolescence and Young Adulthood. Journal of Consulting and Clinical Psychology, 53(6), 890-904. doi: 10.1037/0022-006X.53.6.890

Dziuban, C. D., \& Shirkey, E. C. (1974). When is a correlation matrix appropriate for factor analysis? Some decision rules. Psychological Bulletin, 81(6), 358-361. doi:10.1037/h0036316.

Ferrando, P.J. \& Lorenzo-Seva, U. (2018). Manual Of The Program FACTOR. Recuperado de http://psico.fcep.urv.es/utilitats/factor/index.html.

Forbes, M. K., Kotov, R., Ruggero, C. J., Watson, D., Zimmerman, M., \& Krueger, R. F. (2017). Delineating the joint hierarchical structure of clinical and personality disorders in an outpatient psychiatric sample. Comprehensive Psychiatry, 79, 19-30. doi:10.1016/j.comppsych.2017.04.00

Hair, J. F., Black, W. C., Babin, B. J., Anderson, R. E., \& Tatham, R. L. (2009). Análise multivariada de dados. Bookman Editora.

Hauck Filho, N., Salvador-Silva, R., \& Teixeira, M. A. (2014). Análise de Teoria de Resposta ao Item de um Instrumento Breve de Avaliação de Comportamentos Antissociais. Psico, 45(1), 120-125. Recuperado de http://revistaseletronicas.pucrs.br/ojs/index.php/revistapsico/article/view/14501/1144 8

Jones, S. E., Miller, J. D., \& Lynam, D. R. (2011). Personality, antisocial behavior, and aggression: A meta-analytic review. Journal of Criminal Justice, 39(4), 329-337. doi:10.1016/j.jcrimjus.2011.03.004

Kotov, R., Waszczuk, M. A., Krueger, R. F., Forbes, M. K., Watson, D., Clark, L. A., ... Zimmerman, M. (2017). The hierarchical taxonomy of psychopathology (HiTOP): A dimensional alternative to traditional nosologies. Journal of Abnormal Psychology. doi:10.1037/abn0000258

Kendler, K. S., Aggen, S. H., \& Patrick, C. J. (2012). A multivariate twin study of the DSM-IV criteria for antisocial personality disorder. Biological Psychiatry. doi:10.1016/j.biopsych.2011.05.019

Lobbestael, J., Cima, M., \& Arntz, A. (2013). The relationship between adult reactive and proactive aggression, hostile interpretation bias, and antisocial personality disorder. Journal of Personality Disorders, 27(1), 53-66. doi:10.1521/pedi.2013.27.1.53

Marcus, D. K., Lilienfeld, S. O., Edens, J. F., \& Poythress, N. G. (2006). Is antisocial personality disorder continuous or categorical? A taxometric analysis. Psychological Medicine, 36, 1571-1581. doi: 10.1017/S0033291706008245 
Miller, J. D., \& Lynam, D. (2001). Structural models of personality and their relation to antisocial behavior: A meta-analytic review. Criminology, 39(4), 765-798. doi:10.1111/j.1745-9125.2001.tb00940.x

Moffitt, T. E. (1993). Adolescence-limited and life-course-persistent antisocial behavior: a developmental taxonomy. Psychological Review, 100(4), 674-701. doi:10.1037/0033-295X.100.4.674

Muthén, L. \& Muthén, B. (1998-2012). Mplus User’s Guide. Version 7. Los Angeles, CA: Muth'en \& Muth'en.

Rautiainen, M. R., Paunio, T., Repo-Tiihonen, E., Virkkunen, M., Ollila, H. M., Sulkava, S., Tiihonen, J. (2016). Genome-wide association study of antisocial personality disorder. Translational Psychiatry. doi:10.1038/tp.2016.155

Rosenström, T., Gjerde, L. C., Krueger, R. F., Aggen, S. H., Czajkowski, N. O., Gillespie, N. A., ... Ystrom, E. (2018). Joint factorial structure of psychopathology and personality. Psychological Medicine, 1-10. doi:10.1017/s0033291718002982

Santana, G. L., Coelho, B. M., Wang, Y.-P., Chiavegatto Filho, A. D. P., Viana, M. C., $\&$ Andrade, L. H. (2018). The epidemiology of personality disorders in the Sao Paulo Megacity general population. PLoS ONE, 13(4). doi: 10.1371/journal.pone.0195581

Submetido em: 02/06/2020

Aprovado em: 21/07/2020 